\title{
Use of macular thickness parameters for the diagnosis of primary open-angle glaucoma
}

\author{
Barbara Polaczek-Krupa, Iwona Grabska-Liberek, Michał Kamiński
}

Department of Ophthalmology, Medical Centre of Postgraduate Education, Warsaw, Poland

Submitted: 9 August 2012

Accepted: 6 March 2013

Arch Med Sci 2014; 10, 1: 104-109

DOI: $10.5114 /$ aoms.2014.40736

Copyright $\odot 2014$ Termedia \& Banach

\section{Abstract}

Introduction: Retinal thickness in primary open-angle glaucoma patients was determined to establish its correlation with advancement of glaucoma neuropathy.

Material and methods: One hundred ninety-four patients (371 eyes, age 30-65 years) were divided in 3 groups including 50 patients with confirmed primary open-angle glaucoma, 67 primary open-angle glaucoma-suspected patients and 77 healthy persons (control group). The retinal foveola, foveal, peri-foveal and posterior pole average thickness values were determined with an RTA analyzer. For comparison, linear cup-to-disc diameter ratio $(C / D)$, nerve fiber index (NFI) and mean defect of the retina sensitivity (MD) were also determined.

Results: Statistically highly significant differences in the retinal thickness and glaucoma parameters between the individual groups were observed. In the peri-foveal region, the retinal thickness in glaucoma patients was only $173.0 \pm 11.4 \mu \mathrm{m}$ while in the healthy patients $201.1 \pm 13.1 \mu \mathrm{m}$. In the posterior pole region, the thickness values were only $168.1 \pm 11.3 \mu \mathrm{m}$ and 195.7 $\pm 12.3 \mu \mathrm{m}$, respectively. A moderate correlation between retinal thickness in peri-foveal and posterior pole regions and the C/D, NFI and MD parameters was also established (Pearson coefficients below -0.351 or above 0.284 ).

Conclusions: The retinal thickness in the peri-foveal and posterior pole regions depends on the degree of glaucoma advancement. This original observation may be a basis for acceptance of this method as a quite new tool in glaucoma diagnosis.

Key words: primary open angle glaucoma, retinal thickness, macula, retinal thickness analyzer, retinal thickness analysis.

\section{Introduction}

Modern diagnosis of primary open-angle glaucoma consists in evaluation of the optic disc, neuroretinal rim and retinal nerve fiber layer by indirect stereoscopic observation using the slit lamp and Volk lens [1]. This examination depends on physician experience and can be hardly used because of high variability of optic nerve head structure. Nowadays, laser-scanning ophthalmoscopy (HRT) and laser scanning polarimetry (GDx) are commonly used additional diagnostic methods. The methods are very useful in the early stage of glaucoma; however, direct ophthalmologic examination still remains the basic tool, because results achieved by modern methods are often inefficient for glaucoma diagnosis. This is why other diagnostic methods are also necessary.

\author{
Corresponding author: \\ Iwona Grabska-Liberek \\ Department \\ of Ophthalmology \\ Medical Centre \\ of Postgraduate Education \\ 231 Czerniakowska St \\ 00-416 Warsaw, Poland \\ Phone: +48225841185 \\ E-mail: liberek@gmail.com
}


Retinal thickness analysis (RTA) is a new method in glaucoma diagnosis and has not been used till now. It seems that measurements performed in the perifoveal region can also be sensitive and reliable enough, because the anatomic structure of the scleral channel of the optic nerve does not affect the architecture of the nerve fiber and ganglion cell layers. Changes in the retinal thickness in the macular region can deliver indirect information on atrophy of ganglion cells and nerve fibers [2-4].

The aim of the study was to determine the retinal thickness in primary open-angle glaucoma patients, primary open-angle glaucoma-suspected patients and in healthy persons using the retinal thickness analyzer (RTA) and to establish the correlation between retinal thickness in the macular region and the advancement of glaucoma neuropathy as determined using the linear cup-to-disc diameter ratio (C/D), nerve fiber index (NFI) and mean defect (MD) parameters.

\section{Material and methods}

One hundred ninety-four patients (371 eyes, age 30-65 years) who presented in 2007-2009 at the Department of Ophthalmology of the Medical Centre for Postgraduate Education (CMKP) in Warsaw were included in the study. The patients were divided into 3 groups. Group A consisted of 50 glaucoma patients (95 eyes, average age $43.7 \pm 9.0$ years) with confirmed primary open-angle glaucoma. Group B consisted of 67 primary open-angle glaucoma-suspected patients (128 eyes, average age $43.2 \pm 11.0$ years) without any changes in the visual field. The control group C consisted of 77 healthy persons (148 eyes, average age $46.3 \pm 10.9$ years). The patients were qualified for the particular groups according to directions of the European Glaucoma Society [1] earlier described in detail [5-7]. Characteristics of the studied population are given in Table I.

All patients underwent full ophthalmologic examination. The retinal thickness was determined using the RTA analyzer (Talia Technology Ltd., Israel) after mydriasis with $1 \%$ tropicamide solution. In all groups, foveola average thickness (VAV), foveal average thickness (FAV), peri-foveal average thickness (PFAV) and posterior pole average thickness (PPAV) were determined. Linear cup-todisc diameter ratio (C/D) was determined using a HRT II laser-scanning ophthalmoscope (Heidelberg retina tomograph) made by Heidelberg Engineering. Nerve fiber index was determined using a GDx VCC laser-scanning polarimeter (glaucoma diagnostics with variable corneal compensation) made by Laser Diagnostic Technologies. Mean defect of the retina sensitivity (MD) was determined during visual field examination using frequency doubling technology (FDT). The ophthalmologic examinations and RTA studies were performed in the Ophthalmology Department of the CMKP. The GDx, HRT and FDT studies were carried out at the Institute of Glaucoma and Eye Diseases in Warsaw.

The project received permission from the Bioethics Commission of CMKP on 30 January 2008. The patients were informed of the aim and course of the study and agreed to take part in the project.

\section{Statistical analysis}

The data were statistically evaluated. The significance of differences between the mean values of retinal thickness in the particular regions and mean values of C/D, NFI and MD in particular groups was evaluated using Student's t test. Power of the correlations of VAV, FAV, PFAV and PPAV with $C / D, N F I$ and MD were described with linear Pearson correlation coefficient $[8,9]$.

\section{Results}

Retinal thickness data in particular regions (VAV, FAV, PFAV and PPAV) for the patients in groups $A, B$, and $C$ are presented in Table II. The data concerning $C / D, N F I$ and MD parameters for the patients in groups $A, B$, and $C$ are presented in Table III. In Table IV, linear Pearson correlation coefficients are presented to describe power of the correlations of VAV, FAV, PFAV and PPAV with C/D, NFI and MD. In Table V, linear Pearson correlation coefficients of C/D, NFI and MD are presented for comparison purposes.

The correlations between retinal thickness in particular regions and $C / D, N F I$ and MD parameters in the whole population (A, B, and C groups) are presented in Figures 1-3.

\section{Discussion}

The studied population (Table I) was selected according to directives of the European Glaucoma Society [1] and the individual groups A, B, and C showed comparable sizes and age of the patients included.

Retinal thickness in glaucoma patients and healthy persons, presented in Table II, can be

Table I. The studied population (groups A, B and C) according to sex, number of persons (number of eyes)

\begin{tabular}{|lccc|}
\hline \multirow{2}{*}{ Group } & \multicolumn{2}{c}{ Gender } & Total \\
\cline { 2 - 3 } & Male & Female & \\
\hline A & $27(51)$ & $23(44)$ & $50(95)$ \\
\hline B & $21(40)$ & $46(88)$ & $67(128)$ \\
\hline C & $14(27)$ & $63(121)$ & $77(148)$ \\
\hline Total & $62(118)$ & $132(253)$ & $194(371)$ \\
\hline
\end{tabular}


Table II. Results of determination of retinal thickness in particular VAV, FAV, PFAV and PPAV regions in groups $A, B$ and $C[\mu m]$

\begin{tabular}{|lcccc|}
\hline & VAV & FAV & PFAV & PPAV \\
\hline Group A & & & & \\
\hline Mean value & 139.0 & 149.9 & 173.0 & 168.1 \\
\hline Standard deviation & 20.5 & 17.4 & 11.4 & 11.3 \\
\hline Group B & & & & \\
\hline Mean value & 147.6 & 157.1 & 187.3 & 182.5 \\
\hline Standard deviation & 21.2 & 18.6 & 15.6 & 14.8 \\
\hline Group C (control) & & & & \\
\hline Mean value & 160.9 & 171.2 & 201.0 & 195.7 \\
\hline Standard deviation & 21.4 & 19.8 & 13.1 & 12.3 \\
\hline
\end{tabular}

Table III. Results of determination of C/D, NFI and MD parameters in groups $A, B$ and $C$

\begin{tabular}{|llll|}
\hline \multicolumn{1}{|l}{ C/D } & NFI & MD \\
\hline Group A & & & \\
\hline Mean value & 0.65 & 37.0 & -3.00 \\
\hline Standard deviation & 0.11 & 22.7 & 5.07 \\
\hline Group B & & & \\
\hline Mean value & 0.58 & 18.5 & -0.77 \\
\hline Standard deviation & 0.11 & 5.6 & 2.49 \\
\hline Group C (control) & & & \\
\hline Mean value & 0.43 & 15.1 & -0.29 \\
\hline Standard deviation & 0.11 & 4.8 & 1.94 \\
\hline
\end{tabular}

Table IV. Pearson correlation coefficients between retinal thickness in particular regions and the C/D, NFI and MD parameters in the whole population (groups A, B and C)

\begin{tabular}{|lcccc|}
\hline Parameter & VAV & FAV & PFAV & PPAV \\
\hline C/D & -0.328 (moderate) & -0.324 (moderate) & -0.352 (moderate) & -0.351 (moderate) \\
\hline NFI & -0.109 (weak) & -0.099 (minute) & -0.351 (moderate) & -0.355 (moderate) \\
\hline MD & 0.131 (weak) & 0.119 (weak) & 0.289 (moderate) & 0.284 (moderate) \\
\hline
\end{tabular}

Table V. Pearson correlation coefficients between the NFI, C/D and MD parameters in the whole population (groups A, B and C)

\begin{tabular}{|lcc|}
\hline & C/D & MD \\
\hline NFI & 0.481 (moderate) & -0.400 (moderate) \\
\hline C/D & & -0.400 (moderate) \\
\hline
\end{tabular}

partly compared with the respective literature data. According to the available sources, the average fovea retinal thickness in the healthy persons was $167 \mu \mathrm{m}$ [10], $16.0 \pm 23.0 \mu \mathrm{m}$ [11], 168.57 $\pm 22.27 \mu \mathrm{m}[12]$ and $178 \pm 44 \mu \mathrm{m}$ [13]. The posterior pole average thickness was $229 \mu \mathrm{m}$ [10], $171.9 \pm 25.3 \mu \mathrm{m}$ [14], $175 \pm 14 \mu \mathrm{m}$ [15], 177.77 $\pm 26.13 \mu \mathrm{m}[16]$ and $211.4 \pm 168 \mu \mathrm{m}[4]$ in the same group. The measured and literature data

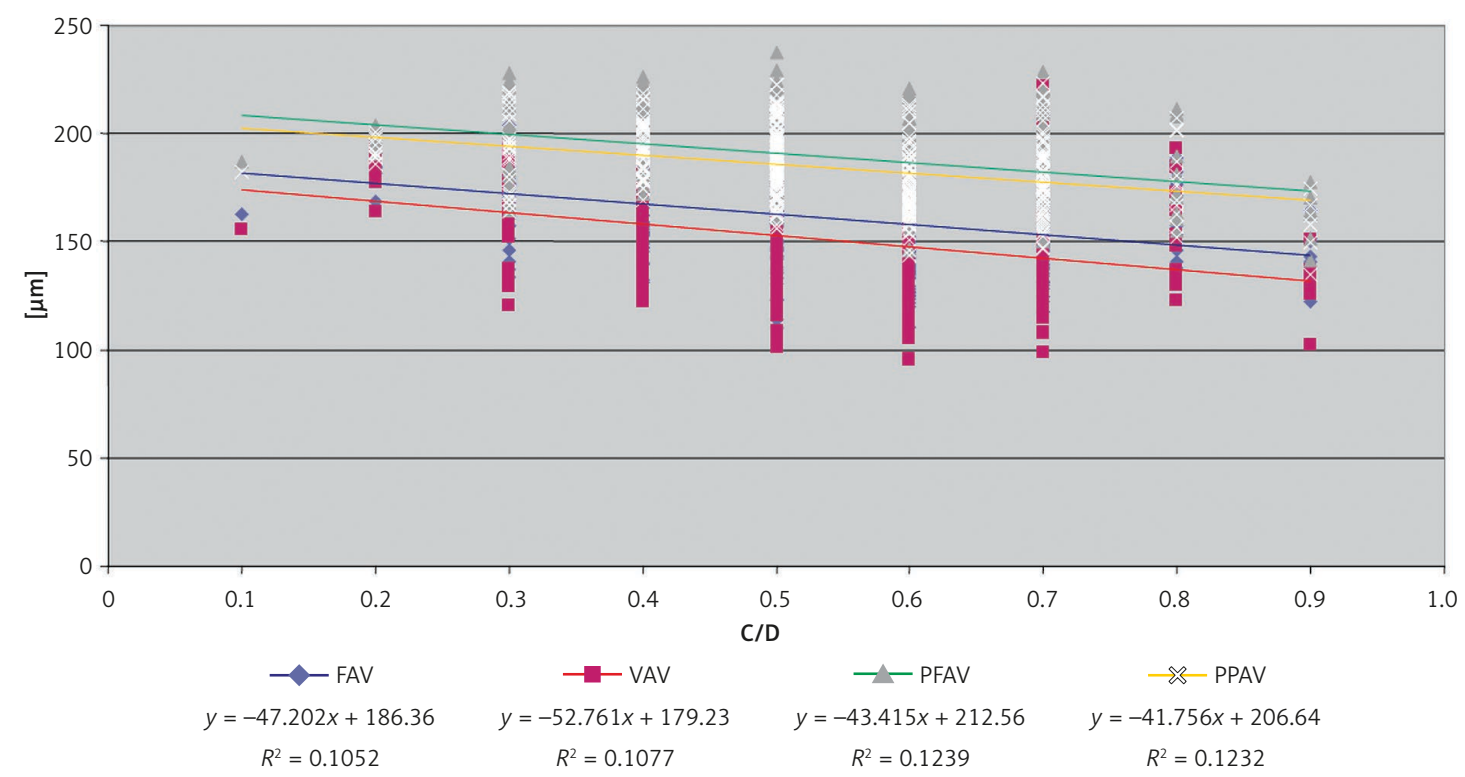

Figure 1. Correlations between retinal thickness in particular regions and the C/D parameter in the whole population (groups A, B and C) 


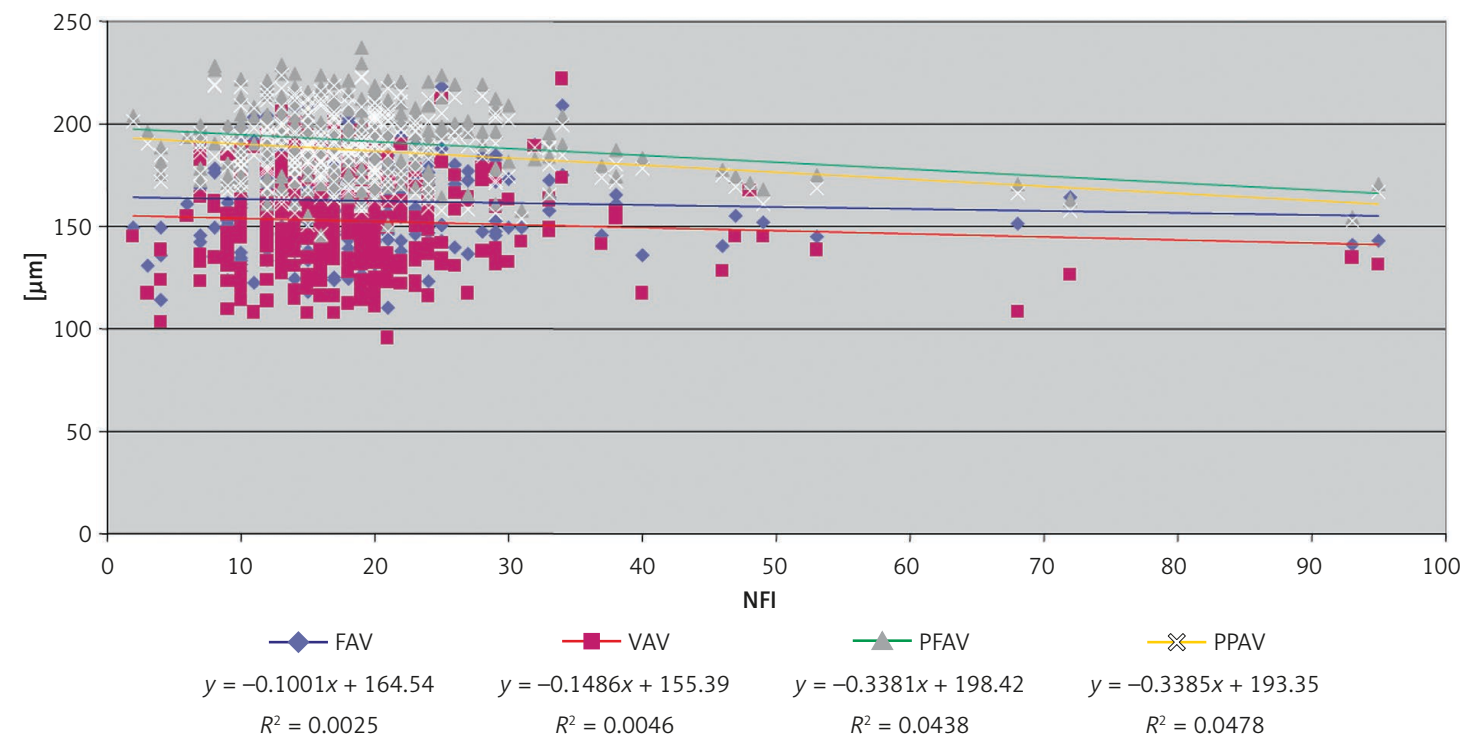

Figure 2. Correlations between retinal thickness in particular regions and the NFI parameter in the whole population (groups A, B and C)

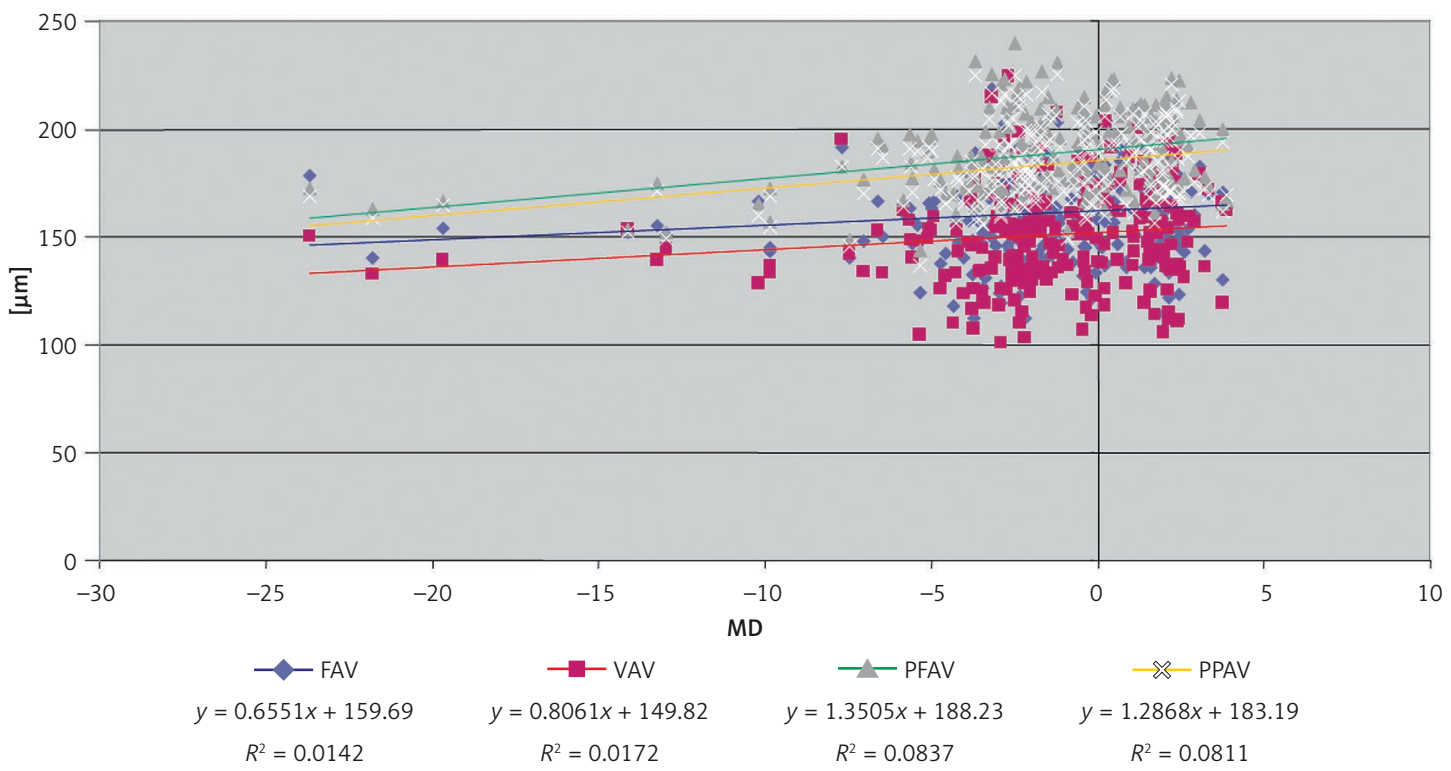

Figure 3. Correlations between retinal thickness in particular regions and the MD parameter in the whole population (groups A, B and C)

are comparable though they show quite high dispersion.

There are available only limited data on the retinal thickness in glaucoma patients. Zeimer et al. [2] examined 18 primary open-angle glaucoma patients but described only 5 of them. The patients also showed a decreased retinal thickness. Similarly, Brusini et al. [3] observed a decrease in retinal thickness in glaucoma patients. Tanito et al. [4] determined the retinal thickness in the perifoveal region as 185.7 $\pm 16.8 \mu \mathrm{m}$ while Zhikuan and Du Shuhua [16] found $152.21 \pm 25.54 \mu \mathrm{m}$. The values differ not only one from another but also from the mea- sured data (Table II). Similarly, limited data are available for glaucoma-suspected patients. Only Tanito et al. [4] have determined the perifoveal retinal thickness: $190.4 \pm 15.6 \mu \mathrm{m}$. This value is very close to the value measured in the present study.

The retinal thickness values in particular groups differed significantly and can be successfully applied in early diagnosis of primary open-angle glaucoma, especially for studying perifoveal and posterior pole regions, where the thinning effects were of a large extent.

The C/D values (Table III) showed, according to common expectations, very large differences be- 
tween particular groups but they can be hardly used for differentiation between groups $A$ and $B$, because of relatively small differences. The NFI values also differ significantly between the individual groups. According to the data published by da Pozzo et al. [17], the NFI index in healthy persons was lower than 18, while in glaucoma patients it exceeds 31 . This agrees with the data measured in the present study. The diagnostic value of the NFI index was also confirmed by Reus and Lemi [18], Zheng et al. [19] and Medeiros et al. [20]. Also, the $M D$ value differed significantly between healthy persons and glaucoma patients. The measured MD values were close to literature data. For example, Bowd et al. [21] found MD $-0.16 \mathrm{~dB}$ for healthy persons and $M D-3.8 \mathrm{~dB}$ for glaucoma patients. Boden et al. [22] found MD $-2.9 \mathrm{~dB}$, and Racette [23] MD -3.69 dB for glaucoma patients. The literature data agree well with the present data also in this area.

The strongest correlation between retinal thickness and C/D (Figure 1), MD (Figure 2) and NFI (Figure 3) parameters was observed in perifoveal and posterior pole regions. In all cases, a decreased retinal thickness was established with increasing values of the C/D, MD and NFI parameters (Figures 1-3). The comparison of the linear Pearson correlation coefficients (Table IV) between retinal thickness in particular regions and $C / D$, $\mathrm{NFI}$ and MD parameters allows for confirmation of substantial relationships between the peri-foveal and posterior-pole retinal thickness and the advancement of glaucoma. There are no data in the literature on this subject. Only Zeimer et al. [2], Tanito et al. [4], Cvenkel et al. [24] and Brusini et al. [3] have observed a connection between the decrease in retinal sensitivity and decrease in retinal thickness in the macular area.

The correlation coefficients between the accepted diagnostic methods (NFI, C/D, MD) are low (Table $\mathrm{V}$ ), which can be explained by high dispersion of the determined values. Therefore, the low values of correlation coefficients between $C / D$, $\mathrm{NFI}, \mathrm{MD}$ and retinal thickness in particular areas (Table IV) are not surprising and do not bring any proof against the retinal thickness analysis.

In conclusion, there is a close relationship between retinal thickness in the macular area and occurrence of glaucoma neuropathy. The retina in the macular region is significantly thinner in primary open-angle glaucoma than in primary open-angle glaucoma-suspected patients and healthy persons.

There is a moderate correlation between retinal thickness in peri-foveal and posterior pole regions and C/D, NFI and MD parameters. The retinal thickness in these regions depends on the degree of glaucoma advancement.
Retinal thickness analysis has not been commonly used for glaucoma diagnosis yet. The measurements carried out in perifoveal and posterior pole regions show a high reproducibility and low standard deviation. This original observation is a basis for acceptance of this method as a quite new tool in glaucoma diagnosis [7].

\section{Acknowledgments}

This study was supported by CMKP, grant 5012-1-14-58/08.

\section{References}

1. Terminology and guidelines for glaucoma. Wyd. European Glaucoma Society, Editrice Dogma S.r.l., Savona (Italy) 2008.

2. Zeimer R, Asrani S, Zhou S, et al. Quantitative detection of glaucomatous damage at the posterior pole by retinal thickness mapping. Ophthalmology 1998; 105: 224-31.

3. Brusini P, Tosoni C, Miani F. Quantitative mapping of the retinal thickness at the posterior pole in chronic open angle glaucoma. Acta Ophthalmol Scan Suppl 2000; 232: $42-4$

4. Tanito M, Itai N, Ohira A, Chihara E. Reduction of posterior pole retinal thickness in glaucoma detected using the retinal thickness analyzer. Ophthalmology 2004; 111: 265-75.

5. Polaczek-Krupa B, Grabska-Liberek I. Changes of the retinal thickness in the macula region in primary open-angle glaucoma patients measured with RTA analyzer [Polish]. Klinika Oczna 2010; 112: 24-8.

6. Polaczek-Krupa B, Grabska-Liberek I. Evaluation of retinal thickness in the macula region in a population of healthy subjects by using the retinal thickness analyzer RTA [Polish]. Klinika Oczna 2009; 111: 217-20.

7. Polaczek-Krupa B. Use of the analysis of retinal thickness in macular region as a new method for diagnostics of primary open-angle glaucoma [Polish]. Thesis, CMKP, Warsaw 2010.

8. Petrie A, Sabin C. Medical statistics in outline [Polish]. PZWL, Warsaw 2006.

9. Stanisz A. Biostatistics [Polish]. Jagiellonian Univ. Press, Cracow 2005; 235-62.

10. Zeimer R. Application of the retinal thickness analyzer to the diagnosis and management of ocular diseases. Ophthalmic Physics Laboratory, Wilmer Eye Institute, Baltimore USA 1998.

11. Zou H, Zhang X, Xu X, Yu S. Quantitative in vivo retinal thickness measurement in Chinese healthy subjects with retinal thickness analyzer. Invest Opthalmol Vis Sci 2006; 47: 341-7.

12. Zhikuan Y, Shuhua D. Histological measurement of human retinal thickness. J Tongji Medical Univ 1999; 19: 246-8.

13. Landau D, Schneidemann EM, Jacobovitz T, Rozenmann Y. Quantitative in vivo retinal thickness measurements in healthy subjects. Ophthalmology 1997; 104: 639-42.

14. Chan CM, Yu JH, Chen LJ, et al. Posterior pole retinal thickness measurements by the retinal thickness analyzer in healthy Chinese subjects. Retina 2006; 26: 176-81.

15. Asrani S, Zou S, d'Anna S, et al. Noninvasive mapping of the normal retinal thickness at the posterior pole. Ophthalmology 1999; 108: 269-73. 
16. Zhikuan Y, Du Shuhua. Mesaurement of retinal thickness in normal subjects with retinal thickness analyzer. J Huazhong Univ Sci Technol 2008; 19: 314-8.

17. Da Pozzo S, Fuser M, Vattovani O, et al. GDx-VCC performance in discriminating normal from glaucoma eyes with early field loss. Graefes Archive for Clinical and Exp. Ophthalmology 2006; 244: 689-95.

18. Reus NJ, Lemii HG. Diagnostic accuracy of the GDx VCC for glaucoma. Ophthalmology 2004; 111: 1860-5.

19. Zheng W, Baohua C, Qun C, et al. Retinal nerve fiber layer images captured by GDx-VCC in early diagnosis of glaucoma. Ophthalmologica 2008; 222: 17-20.

20. Medeiros FA, Bowd C, Zangwill LM, et al. Detection of glaucoma using scanning laser polarimetry with enhanced corneal compensation. Invest Ophthalmol Vis Sci 2007; 48: 3146-53.

21. Bowd C, Zangwill LM, Berry CC, et al. Detecting early glaucoma by assessment of retinal nerve fiber layer thickness and visual function. Invest Ophthalmol Vis Sci 2001; 42: 1993-2003.

22. Boden C, Pascual J, Medeiros FA, et al. Relationship of SITA and full-threshold standard perimetry to frequency-doubling technology perimetry in glaucoma. Invest Ophthalmol Vis Sci 2005; 46: 2433-9.

23. Racette L, Medeiros FA, Zangwill LM, et al. Diagnostic accuracy of the Matrix 24-2 and original N-30 frequency-doubling technology tests compared with standard automated perimetry. Invest Ophthalmol Vis Sci 2008; 49: 954-960.

24. Cvenkel B. Retinal thickness at the posterior pole in glaucoma and ocular hypertension. Graefes Arch Clin Exp Ophthalmol 2004; 241: 920-5. 